\section{Feature saliency and recognition memory for schematic faces*}

\author{
MORTON P. FRIEDMAN, STEPHEN K. REED \\ and \\ EDWARD C. CARTERETTE
}

University of California at Los Angeles, Los Angeles, California 90024

Memory for schematic faces was studied using a recognition memory paradigm. Ss indicated whether two faces, separated by delays of 6,12 , or $18 \mathrm{sec}$, were the same or different. Theoretical interest centered on the effects of component feature saliency on response bias and detectability. The main results were that there were fewer misses and more false alarms on the more salient features. Analysis showed that the main effect of feature saliency was on response bias, not detectability.

Our main concern in this study is with the question of selective memory for the component features of a pattern. In particular, we are interested in whether or not the more salient features of a pattern are better remembered, and how feature saliency interacts with discriminability. To illustrate the distinction we wish to make between saliency and discriminability, consider the stimuli used in the present study. Figure 1 shows two examples of schematic faces which vary in two values of the four component features: forehead height, eye separation, nose length, and mouth height. By the saliency of a feature, we mean the differences in noticeability of a feature with respect to the other features. By the discriminability of a feature, we mean the ability of $S s$ to discriminate between different values of the same feature.

A recognition memory procedure was used in the present study. Ss were shown a schematic face and, after a variable delay, a second face. They judged the second face to be the "same" or "different" from the first face. If they judged the two faces different, they indicated the feature or features of the two faces which differed.

The theoretical problems we are concerned with are best discussed within the framework of a signal detection analysis of memory, as originally introduced by Egan (1958) and since elaborated by many others. [See Kintsch (1970) for a discussion of this work.] According to the signal detection analysis of recognition memory, a $\mathbf{S}$ compares his memory of the features of the first face with his immediate perception of the second face. The differences between memory and perception correspond to the "noise" distribution when the two faces are the same, and the "signal"

* Research supported by National Institute of Mental Health Grant MH-07809. distribution when the two faces are different. We will refer to these distributions as the "same" and "different" distributions. Kinchla and Smyzer (1967) have developed a model for sensory memory based on these notions and have shown that differences in delay between the presentation of the stimuli can be interpreted in terms of increasing distances ( $d$ ") between the "same" and "different" distributions.

Within this framework, how might saliency and discriminability affect memory? One possibility is that the more salient features will show slower memory decay. Thus, the $d^{\prime}$ sctores will be greater for the more salient features. Alternatively, it may be that only response bias is affected by saliency. In this case, saliency would determine only the response criterion $(\beta)$ for saying "same" or "different," and not $d^{\prime}$. The interpretation of discriminability in the above theoretical framework is simply that, given equal rates of decay of memory for features, the more discriminable features should yield larger $d^{\prime}$ scores.

These hypotheses are explored in this study by relating alternative measures of saliency and discriminability to the bias and detectability scores on the four features.

\section{METHOD}

\section{Subjects}

Forty-nine UCLA students served as paid Ss. Ss were paid $\$ 4.00$ for two sessions lasting a total of $2 \mathrm{~h}$. Three Ss were rejected for missing responses or failing to follow instructions.

\section{Stimuli}

Slides of 16 Brunswik schematic faces were used as stimuli. Examples are shown in Fig. 1. The 16 faces were all possible combinations of four features, with each feature having two values. The four features were: (1) the height of the forehead, as determined by the height of the eyes, (2) the distance between the two eyes, (3) the length of the nose, and (4) the height of the mouth. Slides were made from a drawing of a face which was $240 \mathrm{~mm}$ long and $160 \mathrm{~mm}$ wide. The difference between the two values of each feature was always $32 \mathrm{~mm}$. The height of the forehead was either $54 \mathrm{~mm}$ or $86 \mathrm{~mm}$; the distance between the eyes was either $20 \mathrm{~mm}$ or $52 \mathrm{~mm}$; the length of the nose was either $32 \mathrm{~mm}$ or $64 \mathrm{~mm}$; and the height of the mouth was either $28 \mathrm{~mm}$ or $60 \mathrm{~mm}$.

\section{Procedure}

Ss were run in a room which allowed up to eight $S s$ to be run simultaneously. Usually about five Ss were run in one session. Ss received written instructions which stated that the purpose of the experiment was to determine how people remember complex patterns. Examples of the stimuli were shown, and the features which varied were explicitly pointed out.

The experiment utilized a recognition memory paradigm in which Ss indicated whether the second of two faces was the "same" as or "different" from the first face. The faces were projected on the screen for $4 \mathrm{sec}$, and the interval between the two faces was 6,12 , or 18 sec. A 35-mm slide projector was used to present the faces at a visual angle of about $8.5 \mathrm{deg}$. Ss responded by marking prepared answer sheets. The intertrial interval was 6 sec. A pilot study indicated that little forgetting occurred unless Ss performed an intervening task during the retention interval. Therefore, $S s$ canceled three-digit random numbers which were divisible by three during the interval between the two faces.

The first session consisted of three blocks of trials and lasted about $90 \mathrm{~min}$. Ss had a $5-\mathrm{min}$ rest period between each block. A block consisted of 48 trials and included 32 "different" pairs and 16 "same" pairs.
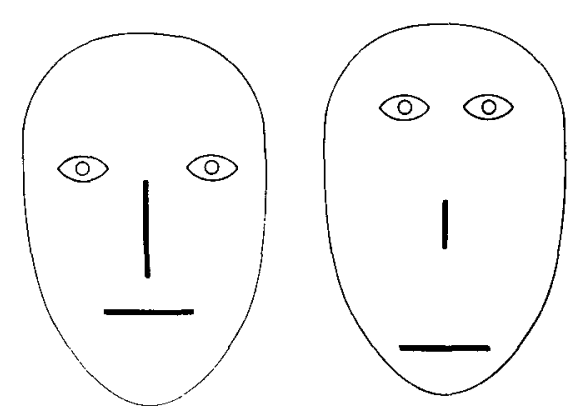

Fig. 1. Examples of the schematic faces used in this research. The faces differ in all features: forehead height, the distance between the eyes, nose length, and height of the mouth. 


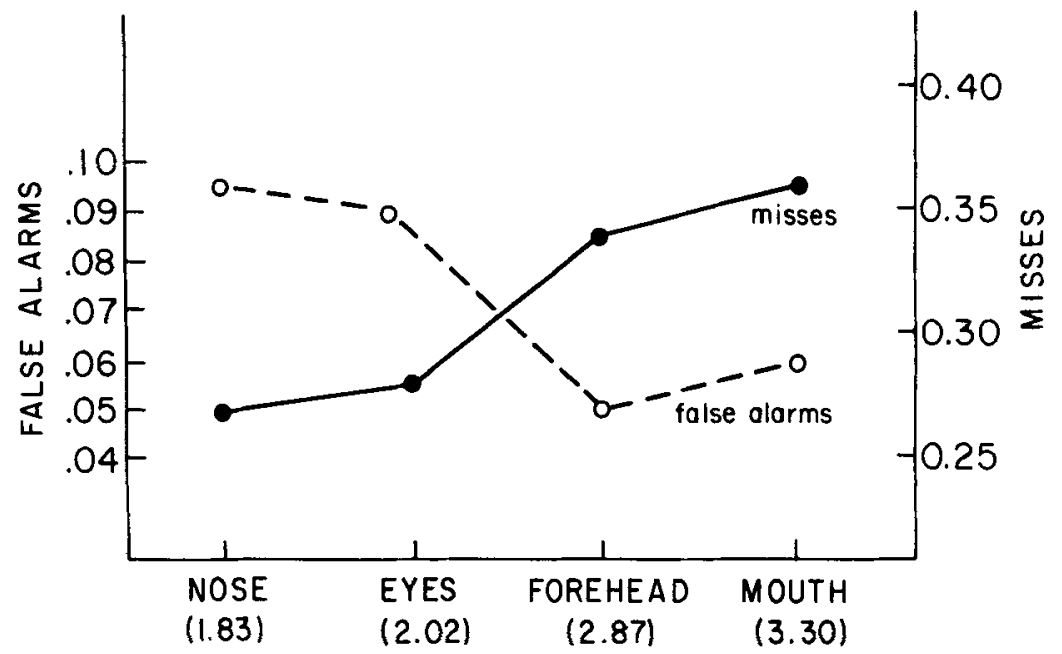

Fig. 2. False alarm and miss errors for the component features. The features are ordered in terms of increasing rank saliency scores. Mean rank saliency scores are given in parentheses for each feature.

Each of the 16 faces was used once as a "same" pair. A "different" pair always differed on only one feature. The 32 "different" pairs were composed of 8 pairs differing on each of the four features. There were 16 delays of 6,12 , and $18 \mathrm{sec}$ in each block. The three delays occurred equally often for each "same" pair and equally often for each of the four features that were varied to produce the "different" pairs. After the experiment, Ss were given the following instruction: "Certain parts of the face stand out and are more salient than others. Rank order the features-forehead, eyes, nose, and mouth - in terms of how noticeable each was." Ss' responses to this question will be referred to as rank saliency.

All Ss returned the following week for the second session. The purpose of the second session was to measure the relative discriminability of the four features and obtain an alternative measure of feature saliency. A procedure used previously by Imai and Garner (1965) was used to measure discriminability. Pictures of the schematic faces were glued on the backs of blank playing cards. A deck of 32 cards, containing two examples of each face, was used. Discriminability of a feature was measured by the speed with which Ss could sort the deck into two piles based on the two values of the feature. Ss first received a practice trial on each of the four features, forming two piles of high and low foreheads, wide and narrow eyes, short and long noses, and high and low mouths. Ss then repeated the procedure for three timed trials. E recorded the total time taken to sort on each feature and ranked the features from the most discriminable (shortest sorting time) to the least discriminable (longest sorting time). This measure will be referred to as the discriminability of features. After $S$ finished the three timed trials on each of the four features, he was asked to rank order the features in terms of discriminability. The rank ordering was very close to the ordering obtained from the sorting times.

The second part of the second session was an attempt to measure feature saliency by using the method of triads. Ss worked through a test booklet containing 48 rows of faces. Each row was composed of three faces, with the left face and right face identical to the center face except for a single feature. Thus, the left face might be identical to the center face except for the nose length. Ss were to decide whether the left or the right face differed more from the center face. Since each face differed on only a single feature, the assumption was that the more salient feature would determine which face differed more from the center face. The four features allowed for six paired comparisons: (1) forehead-eyes, (2) forehead-nose, (3) forehead-mouth, (4) eyes-nose, (5) eyes-mouth, and (6) nose-mouth. Eight judgments were obtained for each of the six pairs. Position effects were balanced by having each member of a pair appear four times on the left and four times on the right. The $\mathrm{E}$ recorded the total number of times each feature was chosen and ranked the features from the one chosen most often (most salient) to the one chosen least often (least salient). This measure will be referred to as choice saliency. This measure confounds saliency and discriminability as defined earlier but is more like the definition of saliency used in the discrimination and concept learning literature, where it is assumed that increasing the discriminability of the values of a feature leads to increased saliency of that feature. This literature is reviewed by Trabasso and Bower (1968).

\section{RESULTS}

Two types of errors were calculated for each of the four features. A "miss" was recorded when a feature changed and $S$ failed to report it. A "false alarm" was recorded when $S$ incorrectly reported that a feature changed. For example, if the two faces differed on mouth height and $S$ reported a difference on nose length, a false alarm was scored for nose and a miss was scored for mouth. Figure 2 shows the average probability of a miss and false alarm for each of the four features. The features are ordered in terms of rank saliency, and the mean rank is shown below each feature. The results show a significant Error by Feature interaction ( $F=13.89$, df $=3 / 135, p<.01$ ), in which the probability of a miss was higher on the features rated low on saliency and the probability of a false alarm was higher on the features rated high on saliency.

The Error by Feature interaction suggests that the saliency of a feature might affect $S$ 's response bias more than his sensory memory for that feature. To test this hypothesis, memory $\left(d^{\prime}\right)$ and bias, or criterion $(\beta)$, parameters were calculated for each of the four features and $46 \mathrm{Ss}$, combining the three delays. Parameter values were obtained for both the theory of signal detectability outlined earlier and a "high threshold theory" [See Friedman, Carterette, Nakatani, \& Ahumada (1968) for an application of high threshold theory to detection and the discussion in Kintsch (1970) on applications to recognition memory.] According to high threshold theory, S correctly perceives a stimulus as the "same" or "different" with probability $s$. If $S$ is uncertain about the stimulus, he biases his response and guesses "different" with probability g, and "same" with probability $1-\mathrm{g}$. Table 1 contains the mean values of these parameters and the results of the $F$ tests comparing the parameter values for the various features. For both theories, it may be seen that the differences among the values for memory parameters were not reliable, but there were significant differences among the bias parameter scores, with the most salient features showing the most bias. These analyses suggest that the main effect of saliency is on response bias, not sensory memory.

Further analyses were done comparing $d^{\prime}$ and $\beta$ scores for the 
Table 1

Memory (s, $d^{\prime}$ ) and Bias $(g, \beta)$ Parameters for Each Feature

\begin{tabular}{lcccc}
\hline & \multicolumn{2}{c}{ Threshold } & Theory & \multicolumn{2}{c}{$\begin{array}{c}\text { Theory of } \\
\text { Signal Detectability }\end{array}$} \\
\cline { 2 - 4 } Features & $\mathrm{s}$ & $\mathrm{g}$ & $\mathrm{d}$ & $\beta$ \\
\hline Nose & 0.63 & 0.29 & 2.27 & 1.68 \\
Eyes & 0.62 & 0.31 & 2.21 & 1.52 \\
Forehead & 0.61 & 0.17 & 2.33 & 2.06 \\
Mouth & 0.58 & 0.21 & 2.14 & 1.77 \\
& $F=1.47$ & $\mathrm{~F}=4.88^{*}$ & $\mathrm{~F}=1.12$ & $\mathrm{~F}=6.13^{*}$ \\
\hline
\end{tabular}

$* p<.01, d f=3 / 135$

various features as a function of delay. These analyses yielded only the expected decrease in $\mathrm{d}^{\prime}$ as a function of increasing delay but no marked differences among the features.

An alternative set of analyses concerned with how the two saliency measures and the discriminability measure were related to the type of errors Ss made on the different features was also run. In these analyses, responses were rescored according to saliency and discriminability measures. For example, in order to test for the effects of ranked saliency, each S's responses were divided into four groups, with the first group containing S's error score on the feature he ranked as the most salient, etc. In scoring the errors, a distinction was made between two types of false alarms. The first type will be referred to as a "false positive," which occurred when $S$ reported that a feature changed when all features remained the same. The second type will be called a "confusion" and indicates that $S$ reported that a particular feature changed when one of the other features actually changed. A confusion error indicates that $S$ is correct in stating that the two faces are different, but he is incorrect as to which feature changed. For each feature, there were 24 trials on which that feature changed, 72 trials on which one of the other three features changed, and 48 trials on which no feature changed. Thus, there could be a maximum of 24 misses, 72 confusions, and 48 false positives per feature. The mean error rate was $32 \%$ for misses, $8 \%$ for confusions, and $6 \%$ for false positives.

Table 2 shows the three types of errors, the memory parameters, and the bias parameters when the features are ordered according to ranked saliency, choice saliency, and discriminability. The combined false alarms were used to calculate the memory and bias parameters.

The variable which had the greatest influence was ranked saliency. The number of confusions increased from 4.00 on the least salient feature to 8.04 on the most salient feature
$(F=10.42, \mathrm{df}=3 / 135, \mathrm{p}<.01)$, while the number of misses declined from 9.76 on the least salient feature to 6.70 on the most salient feature $(F=10.87, \quad d f=3 / 135, \quad p<.01)$. There was also a significant difference in the $d^{f}$ scores $(F=5.84, d f=3 / 135$, $p<.01)$. However, there was little difference in memory among the three most salient features. The feature ranked second in saliency was remembered best, and the features ranked 1 and 3 were remembered about equally well. The criterion $(\beta)$ scores also showed a response bias trend, but the results were not significant, probably as a result of the increased variability resulting from the transformation of low error probabilities into $\mathrm{z}$ scores. For the threshold theory analyses, the bias parameters differed significantly when the features were ordered according to ranked saliency $(\mathrm{F}=5.20, \mathrm{df}=3 / 135$, $p<.01$ ). The bias parameter, which for this model is the probability of guessing that a feature changed, was .33 for the most salient feature, with declining values of $.26, .22$, and .17 as the features became less salient.

Errors, Memory Parameters, and Bias Parameters for the Features Grouped According to Rank Saliency, Choice Saliency, and Discriminability

\begin{tabular}{|c|c|c|c|c|c|c|c|}
\hline & \multirow{2}{*}{$\begin{array}{c}\text { Confu- } \\
\text { sions }\end{array}$} & \multirow{2}{*}{$\begin{array}{l}\text { False } \\
\text { Positives }\end{array}$} & \multirow[b]{2}{*}{ Misses } & \multicolumn{2}{|c|}{$\begin{array}{c}\text { Theory of Signal } \\
\text { Detectability }\end{array}$} & \multicolumn{2}{|c|}{$\begin{array}{l}\text { Threshold } \\
\text { Theory }\end{array}$} \\
\hline & & & & $d^{\prime}$ & $\beta$ & $\mathbf{s}$ & $\mathbf{g}$ \\
\hline \multicolumn{8}{|c|}{ Rank Saliency } \\
\hline 1 & 8.04 & 3.04 & 6.70 & 2.24 & 1.61 & 0.63 & 0.33 \\
\hline 2 & 6.54 & 2.72 & 6.33 & 2.42 & 1.77 & 0.66 & 0.26 \\
\hline 3 & 5.20 & 2.96 & 7.54 & 2.26 & 1.84 & 0.63 & 0.22 \\
\hline 4 & 4.00 & 3.13 & 9.76 & 2.01 & 1.83 & 0.53 & 0.17 \\
\hline $\mathbf{F}$ & $10.42^{*}$ & 0.32 & $10.87^{*}$ & $5.84 *$ & 1.13 & $10.13^{*}$ & 5.20* \\
\hline \multicolumn{8}{|c|}{ Choice Saliency } \\
\hline 1 & 5.07 & 2.63 & 6.89 & 2.40 & 1.94 & 0.64 & 0.24 \\
\hline 2 & 5.54 & 3.11 & 8.37 & 2.19 & 1.75 & 0.60 & 0.26 \\
\hline 3 & 6.07 & 2.89 & 8.04 & 2.13 & 1.70 & 0.59 & 0.26 \\
\hline 4 & 7.11 & 3.22 & 7.02 & 2.22 & 1.67 & 0.61 & 0.25 \\
\hline $\mathbf{F}$ & 2.25 & 0.69 & 2.09 & 2.66 & 1.58 & 1.61 & 0.14 \\
\hline \multicolumn{8}{|c|}{ Discriminability } \\
\hline 1 & 6.61 & 3.07 & 6.15 & 2.38 & 1.86 & 0.66 & 0.26 \\
\hline 2 & 6.13 & 3.50 & 7.78 & 2.23 & 1.60 & 0.59 & 0.29 \\
\hline 3 & 5.65 & 2.57 & 8.15 & 2.13 & 1.86 & 0.59 & 0.20 \\
\hline 4 & 5.39 & 2.72 & 8.24 & 2.19 & 1.72 & 0.59 & 0.24 \\
\hline$F$ & 0.82 & 1.81 & 3.79 & 2.17 & 1.66 & 3.07 & 1.53 \\
\hline
\end{tabular}

$* p<.01, d f=3 / 135$ for all $F$ ratios
Differences among the memory parameter scores for the threshold theory were also significant.

When the features were ordered according to discriminability, there were only two trends of note, both nonsignificant. The number of misses showed a decline with increasing discriminability, and the $\mathrm{d}^{\prime}$ scores were an increasing function of discriminability.

The ranked saliency scores based on the method of triads yielded no significant differences among the features for any of the scores. The only trend worth noting is that confusions decreased as a function of increasing saliency.

\section{DISCUSSION}

The main result of the experiment was that the Ss showed a strong response bias on those features which they had ranked high on saliency. As a result of this response bias, Ss made significantly more confusions and fewer misses on the more salient features. The effect of saliency on the sensory memory for the features is less clear. Although there were significant differences among the memory parameters, this was mainly due to the poor memory for the least salient feature. The feature ranked second in saliency was remembered best and the features ranked first and third on saliency were remembered equally well.

Une finding that merits further discussion is the differences obtained for the two types of false alarms. The 
higher false alarm rate on the more salient features was due entirely to the greater number of confusions made on the more salient features. Response bias showed a substantial effect when the two faces were different, but no effect when the two faces were the same. This finding suggests that Ss may have perceived that the two faces were different, but were not sure which feature or features changed. In such cases, their guess tended to be one of the more salient features. This result has important implications for models of pattern memory and for theories of detection and recognition. The issue is whether or not detection of a difference between the two faces implies recognition of the feature that is actually different. Shipley (1965) has reported some work dealing directly with this issue. Her results indicate that, for a simple auditory detection and recognition situation, detection of a signal implies recognition of the identity of that signal. Results in this experiment appear to be at variance with Shipley's results and more in agreement with results of Lindner (1968) for auditory recognition. Bower (1967) has discussed the problem of multidimensional stimulus recognition within the context of his multicomponent theory of memory. He shows that there are a variety of possible rules for combining information from the comparison of the various features of the two patterns. Different rules would yield both results such as we obtained and those obtained by Shipley, and there is simply not enough data available to choose among the alternatives. This is certainly a problem that deserves further work.

The finding that rank saliency resulted in a number of reliable effects and choice saliency resulted in no significant effects raises the question of what is a good operational definition and measure of saliency. Our use of the choice saliency task was an attempt to obtain a more independent behavioral measure of saliency than the ranking obtained from Ss' verbal reports, and to relate this work to previous work in concept learning. The rank saliency measure, for example, may merely reflect that Ss were aware of their response bias and, when asked to rank order the features in terms of which features stood out or were more noticeable, did so on the basis of their response bias.

The small effects attributable to discriminability obviously does not mean that discriminability of the features is an unimportant variable in such experiments. One reliable effect was found-a decline in the number of misses with increasing discriminability and more effects should be observed if discriminability were varied to a greater extent.

One final remark concerns our identification of the "different" feature distribution with the signal distribution. In general, we believe that whatever $\mathbf{S}$ is trying to detect and report in his response should be represented by the signal distribution. In a signal-detection experiment, he reports signals; in this experiment, he reported what feature or features changed. In typical recognition experiments, based on single component items, he reports "old" or "new," and it is convenient to label the decision axis memory strength and to represent the old items by the signal distribution. The converse of our experiment would be to present Ss with two faces that were the same on only one feature. S's task would be to report what feature remained the same. An interesting question is whether Ss would continue to respond with a response bias for change in the features, as was the case in the present experiment, or whether a response bias would result in their reporting that the more highly salient features remained the same.

\section{REFERENCES}

BOWER, G. A multicomponent theory of the memory trace. In $\mathrm{K}$. Spence and $\mathrm{J}$. Spence (Eds.), The psychology of learning and motivation: Advances in research and theory. Vol. 1. New York: Academic Press, 1967. Pp. 299-325.

EGAN, J. P. Recognition memory and the operating characteristic. Technical Note AFCRC-TN-58-51, 1958, Indiana University Hearing and Communication Laboratory.

FRIEDMAN, M. P., CARTERETTE, E, C. NAKATANI， L., \& AHUMADA, A. Comparison of some learning models for response bias in signal detection. Perception \& Psychophysics, 1968, 3 . 5-11.

IMAI, S., \& GARNER, W. Discriminability and preference for attributes in free and constrained classification. Journal of Experimental Psychology, 1965, 69, 596-608.

KINCHLA, R. A., \& SMYZER, F. A. A diffusion model of perceptual memory. Perception \& Psychophysics, 1967, 2 219-229.

KINTSCH, W. Learning, memory, and conceptual processes. New York: Wiley, 1970.

LINDNER, W. Recognition performance as a function of detection criterion in a simultaneous detection-recognition task. Journal of the Acoustical Society of America, 1968, 44, 204-211.

SHIPLEY, E. Detection and recognition: Expeximents and choice models. Journal of mathematical psychology, 1965, 2, 277-311.

TRABASSO, T., \& BOWER, G. Attention in learning. New York: Wiley, 1968.

(Accepted for publication January 10, 1971.) 